\title{
Gouy phase compensation in quasi phase matching
}

\author{
Huw E. Major, ${ }^{*}$ Corin B.E. Gawith, and Peter G.R. Smith \\ Optoelectronics Research Centre, University of Southampton, UK
}

(Dated: August 6, 2007)

\begin{abstract}
In any focussed nonlinear interaction the focus induced phase shift, known as the Gouy phase shift, provides an imperfection in phase matching for any linearly invariant material. However, using an appropriately designed quasi-phase matched structure it is theoretically possible to completely compensate for the deleterious effects of the Gouy phase shift, allowing a symmetric frequency response and tighter focussing for higher conversion efficiencies.
\end{abstract}

Nonlinear optics provide an essential source of laser light at wavelengths that are otherwise difficult to obtain. These wavelengths can be created with nonlinear effects such as second harmonic generation (SHG) [1, 2], sum and difference frequency generation and optical parametric oscillation (OPO) [3]. The conversion efficiency of such processes are highly dependent on the fundamental laser power, with harmonic output generally increasing quadratically with the fundamental intensity. The large electric fields required for efficient operation normally require focusing to a tight waist.

The effects of focussing laser beams, in particular Gaussian beams, on nonlinear parametric interactions have been examined analytically by Boyd and Kleinman [4] (BK). By analysing the interactions of fundamental and harmonic beams in nonlinear materials under the assumption of no source depletion, they were able to calculate optimal focussing conditions of the laser to give maximal conversion efficiency. This can be interpreted as a balance between tight focusing to give a high intensity beam waist and the need to utilise as much interaction length as possible, with the optimal focussing condition given as

$$
\xi=\frac{L}{b}=\frac{L}{2 z_{R}}=2.84,
$$

where $\mathrm{L}$ is the nonlinear material length, $\mathrm{b}$ is the confocal parameter and $z_{R}=\pi \omega_{o}^{2} n / \lambda$ is the Rayleigh range.

However, as we will show, this particular condition applies only to a linearly invariant crystal where crystal length and Gouy phase shift are both compensated for by compromises in the beam focus.

Here, the Gouy phase shift $[5,6]$ is an inevitable consequence of beam focusing and which limits nonlinear conversion efficiency by preventing perfect phase matching. Its influence can be seen experimentally as an alteration in phase matching conditions between the planewave and optimal focused case, and is most commonly seen as a small temperature or angle tuning shift and increasing asymmetry with tighter focus.

In this paper we shall theoretically demonstrate that the domain reversal techniques of quasi-phase matching (QPM) can totally compensate for the phase error resulting from the Gouy phase shift. Our QPM grating designs, which are no longer linearly invariant, allow for higher efficiencies than can be obtained with standard QPM and furthermore require no shift in phase matching under focused conditions when compared to a plane wave. By carrying out an analysis based on the BK approach we are thus able to predict an optimal quasi-phase matching structure and calculate its performance.

In our initial study we consider the Gouy phase shift as it relates to Gaussian beams. Under focussing a single mode Gaussian beam receives a $\pi$ phase shift to its wave fronts as it travels from $-\infty$ to $\infty$ through a focussed waist. The full expression for the phase variation is given as

$$
\Phi(z)=(m+n+1) \arctan \left(\frac{z}{z_{R}}\right),
$$

where $m$ and $n$ reference the Hermite-Gaussian modes $\left(T E M_{m n}\right)$.

In the case of SHG both the fundamental and harmonic waves must propagate in phase to obtain coherent energy transfer into the second harmonic. For the simple plane wave case efficient phase matching can be achieved by either birefringent phase matching in an appropriate material or by QPM in materials such as periodically poled lithium niobate (PPLN). However, in focussed systems the Gouy phase shift changes the linear phase mismatch relationship of the plane wave to become more complex, containing both the linear (dispersive) part and the Gouy phase for which standard critical and quasi phase matching cannot compensate.

Figure (1a) shows the relative Gouy phase throughout a device when focussed at the BK optimal value. Also shown is the linear phase difference between the coherence length of a device operating under plane wave and the coherence length that provides maximum conversion efficiency at this focus. Device efficiency is maximised by minimising the overall phase error. At very loose focus the Gouy phase variation is approximately linear over the device length and is almost completely compensated for by the linear phase of the device, resulting in minimal phase error as shown in Fig. (1b) curve (i).

However, as the focus is tightened the Gouy phase variation becomes more significant along the device length. By altering the temperature or angle of the nonlinear device a linear phase variation in the coherence length 


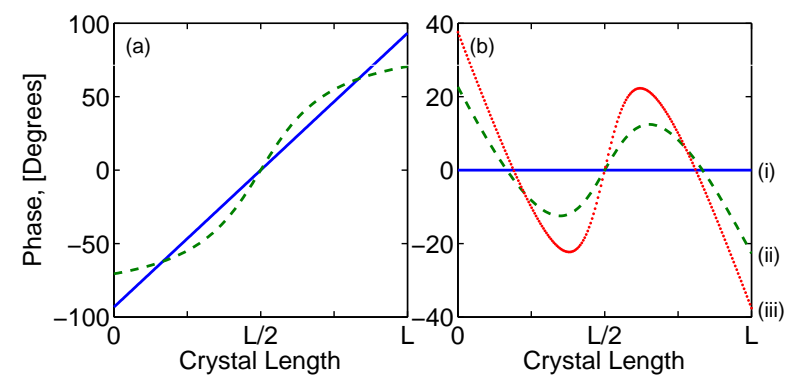

FIG. 1: (a) Gouy phase and corresponding linear phase for optimal operation at $\xi=2.84$, and (b) the resulting phase error between Gouy and linear device phase for focussing at (i) $\xi=0.01$, (ii) $\xi=2.84$, (iii) $\xi=5.0$.

occurs (compared to the plane wave case), which partially compensates for the Gouy phase shift. However it is clear from Fig. (1b) in curves (ii) and (iii) that a significant phase mismatch remains which results in a reduction in maximum conversion efficiency. The focus value of $\xi=2.84$ highlighted by BK provides an operational compromise for phase matching of a focussed beam by balancing the power density advantage against a linear fit to the Gouy phase shift.

We shall now show, by extending the analysis of BK, how a modified QPM grating can be used to completely compensate for the deleterious effects of the Gouy phase shift, leading to higher conversion efficiencies. In this analysis we reproduce the steps of the BK derivation emphasising the key role of the Gouy phase shift on frequency conversion.

Starting with the Gaussian wave equation for a single mode $\mathrm{TEM}_{00}$ beam

$$
E_{1}(r, z, t)=\frac{E_{o}}{2} \frac{\omega_{o}}{\omega(z)} e^{\frac{-r^{2}}{\omega^{2}(z)}} e^{i(k z-\omega t)} e^{i \Psi(z)} e^{i \Phi(z)}+c . c .
$$

where $\omega(z)$ is position dependent beam diameter given by

$$
\omega(z)=\omega_{o}\left[1+\left(\frac{\lambda z}{\pi \omega_{o}^{2} n}\right)^{2}\right]^{1 / 2}
$$

$\Psi$ is the radius of curvature of phase fronts given by

$$
\Psi(z)=\frac{k r^{2}}{2 z\left[1+\left(\frac{\lambda z}{\pi n \omega_{o}^{2}}\right)\right]^{2}}
$$

and $\Phi$ is defined in Eq. (2) with $m=n=0$. Substituting Eq. $(2,4,5)$ into Eq. $(3)$ we have

$$
\begin{aligned}
E_{1}(r, z) & =\frac{E_{o}}{2} \frac{1}{\sqrt{1+\zeta^{2}}} e^{i k_{1} z} e^{\frac{-r^{2}}{\omega_{o}^{2}\left(1+\zeta^{2}\right)}} e^{\frac{i \zeta r^{2}}{\omega_{o}^{2}\left(1+\zeta^{2}\right)}} e^{i \Phi(z)} \\
& =\frac{E_{o}}{2} \frac{1}{1-i \zeta} e^{i k_{1} z} e^{\frac{-r^{2}}{\omega_{o}^{2}(1+i \zeta)}}
\end{aligned}
$$

where $\zeta=z / z_{R}$. From this we determine the induced polarisation of the nonlinear material

$$
\begin{aligned}
P\left(\omega_{2}\right) & =2 \epsilon_{o} d_{\text {eff }}\left|E_{1}(r, z)\right|^{2} \\
& =2 \epsilon_{o} d_{\text {eff }} \frac{E_{o}^{2}}{4}\left(\frac{1}{1-i \zeta}\right)^{2} e^{i 2 k_{1} z} e^{\frac{-2 r^{2}}{\omega_{o}^{2}(1+i \zeta)}}
\end{aligned}
$$

where $d_{\text {eff }}$ is the effective nonlinearity of the material. From this an expression for the resulting harmonic can be realised, in the form

$$
E_{2}(r, z)=A_{2}(r, z) e^{i k_{2} z} .
$$

By considering a slice of material of thickness $d z$ the incremental harmonic is given as

$$
\begin{aligned}
d A_{2}(r, z)= & \frac{-i k_{2}}{2 \epsilon_{o} n_{2}^{2}} e^{i k_{2} z} P\left(\omega_{2}\right) \\
= & \frac{-i k_{2}}{2 \epsilon_{o} n_{2}^{2}} e^{i k_{2} z} 2 \epsilon_{o} d_{e f f} \frac{E_{o}^{2}}{4}\left(\frac{1}{1-i \zeta}\right) \\
& \times\left\{\left(\frac{1}{1-i \zeta}\right) e^{i 2 k_{1} z} e^{\frac{-2 r^{2}}{\omega_{o}^{2}(1+i \zeta)}}\right\} .
\end{aligned}
$$

We notice that the bracketed expression is simply the form of the Gaussian wave equation. Further, by nature of the second harmonic process the Rayleigh range of the SHG Gaussian is equal to that of the fundamental field, resulting in a matching Gouy phase shift.

Following BK, integrating over all sources within the crystal gives the total harmonic field contribution at a point $\left(r^{\prime}, z^{\prime}\right)$ outside the crystal. We further make the substitution of $\Delta k=2 k_{1}-k_{2}$, which provides the phase matching condition for SHG, and define $\zeta=2(z-f) / b$ to allow for arbitrary focal position. Additionally, with the change of variables $\xi=L / b, \mu=(L-2 f) / L$ and $\sigma=\Delta k b / 2$ we arrive at a solution for SHG conversion efficiency under arbitrary focussing conditions, still assuming no pump depletion, to give

$$
\begin{aligned}
E_{2}\left(r^{\prime}, z^{\prime}\right)= & \frac{-i k_{2}}{4 n_{2}^{2}} d_{e f f} E_{o}^{2} \frac{1}{1-i \zeta^{\prime}} e^{\frac{-2 r^{2}}{\omega_{\sigma^{\prime} \zeta^{\prime}\left(1+i \zeta^{\prime}\right)}}} \\
& \times \frac{b}{2} e^{i \Delta k f} \int_{-\xi(1-\mu)}^{\xi(1+\mu)} \frac{e^{i \zeta \sigma}}{1-i \zeta} d \zeta .
\end{aligned}
$$

Eq. (10) provides the key result of the BK analysis. The optimal focusing condition $\xi=2.84$ is that which maximises the integral, firstly by placing the beam waist in the centre of the crystal, but also by selecting a value for $\Delta k$ that provides the best linear compensation in the phase term of the integral for the Gouy phase shift. In the remainder of this paper we will show that by designing a QPM structure with Gouy phase compensation we can promote perfect phase matching for a given focussing condition. We will do this by first introducing a spatially varying nonlinearity to represent quasi-phase matching and secondly introducing an additional phase term that 
varies the period locally to compensate for the focus induced phase shift.

If we now replace the constant $d_{\text {eff }}$ of Eq. (10) with a spatially varying effective nonlinearity, $\kappa(\zeta)$ we can define the equation

$$
H(\sigma, \xi, \mu)=\int_{-\xi(1-\mu)}^{\xi(1+\mu)} \frac{\kappa(\zeta) e^{i \zeta \sigma}}{1-i \zeta} d \zeta
$$

which is comparable to Eq. (2.16) in [4]. From Eq. (11) an expression for $\kappa(\zeta)$ can be obtained which maximises $H(\sigma, \xi, \mu)$ and thus the SHG conversion efficiency. In the case of a plane wave, as assumed for loose focussing, this expression simplifies to the integral of the sinusoidally varying second harmonic electric field along the crystal length. Using standard QPM this integral can be maximised by inverting the material nonlinearity at a period defined by the material dispersion to provide continuous growth of the second harmonic. This can be expressed mathematically as

$$
\kappa(\zeta)=\frac{\cos (\Delta k z)}{|\cos (\Delta k z)|}
$$

where $\kappa(\zeta)= \pm 1$ to reflect the fact that the nonlinearity can take just two values $(+d$ or $-d)$ with periodic poling. The structure defined by Eq. (12), which is a conventional first-order QPM grating, is routinely used in periodically poled materials. Numerical analysis shows it has the same optimal focusing condition as found by BK.

For the more complex case with focussing, Eq. (12) does not account for the Gouy phase slippage. We will now show how a suitable modification to the simple linearly periodic structure allows for compensation of this effect.

By re-arranging the integrand of Eq. (11) and noting that the denominator is in the form of the Gouy phase shift, we obtain

$$
\begin{aligned}
\frac{\kappa(\zeta) e^{i \zeta \sigma}}{1-i \zeta} & =\kappa(\zeta) e^{i \zeta \sigma}\left(\frac{1+i \zeta}{1+\zeta^{2}}\right) \\
& =\frac{\kappa(\zeta) e^{i \zeta \sigma}}{\sqrt{1+\zeta^{2}}} e^{i \arctan (\zeta)}
\end{aligned}
$$

We now wish to identify the optimal design of periodic structure $\kappa(\zeta)$ to maximise the second harmonic efficiency. If we select

$$
\kappa(\zeta)=\frac{\cos (\zeta \sigma)}{|\cos (\zeta \sigma)|} e^{-i \arctan (\zeta)}
$$

the unwanted Gouy phase term is thus negated promoting perfect phase matching. By considering the complex conjugate term throughout the analysis, as is standard in nonlinear optical derivations, Eq. (14) can be simplified to

$$
\kappa(\zeta)=\frac{\cos (\zeta \sigma+\arctan [\zeta])}{|\cos (\zeta \sigma+\arctan [\zeta])|}
$$

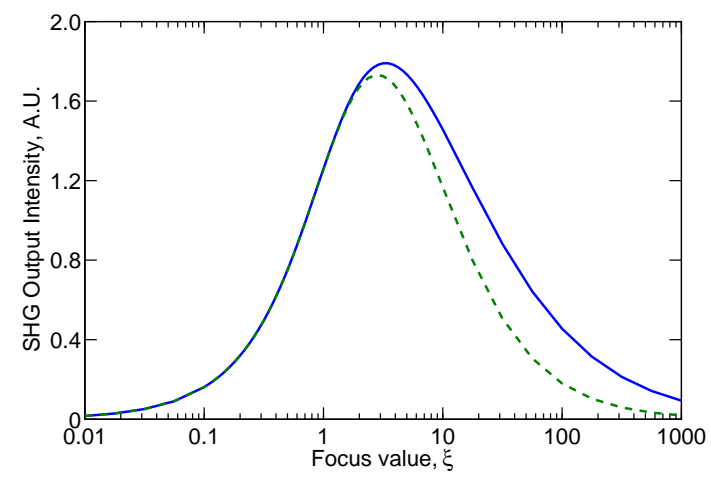

FIG. 2: A graph of second harmonic output intensity as a function of the focus parameter $\xi$ for a standard QPM device (dashed curve) and an equal length of a Gouy phase shift compensated QPM device (solid curve).

In the focussed case it can be seen from Eq. (15) that an additional spatially varying phase correction of $\arctan (\zeta)$ can be introduced as a continuous change in the phase matching period. This addition to the linear case allows our new grating to completely compensate for the effects of the Gouy phase shift. Thus, for a QPM device with Gouy phase compensation the placement of inverted domains is simply described by Eq. (15), with the value of $\sigma$ determined by the material dispersion.

The most prominent result of removing the Gouy phase shift in nonlinear devices is the increase in conversion efficiency that can be achieved. Under any focussing condition, if the QPM grating is suitably compensated efficiencies greater than that produced using standard phase matching techniques can always be obtained. This is especially true as the focus parameter is increased towards the value outlined by BK, where a clear increase in efficiency over QPM can be observed. This effect is illustrated in the graph of Fig. (2), where the curve has been calculated by numerical integration. Furthermore, after this adjustment the peak conversion efficiency is no longer found at the BK focussing value of $\xi=2.84$ but at a more tightly focussed $\xi=3.317$, resulting in a $3.5 \%$ increase in efficiency over the previous best case.

Results of our simulation also show that for values larger than $\xi=2.84$ in a linearly invariant crystal the reduction in efficiency due to the Gouy phase shift outweighs the gain in average power density obtained by tighter focussing, resulting in maximum efficiency at a non-optimal power density. However, with a Gouy phase compensated device, the dominant effect on efficiency is now only the average power density, with the maximum average power obtained at the tighter focus of $\xi=3.317$. As such, to obtain the maximum possible SHG conversion, QPM devices should be fabricated with a grating pitch variation as described by Eq. (15). For the remainder of this paper we shall refer to such an a device as 


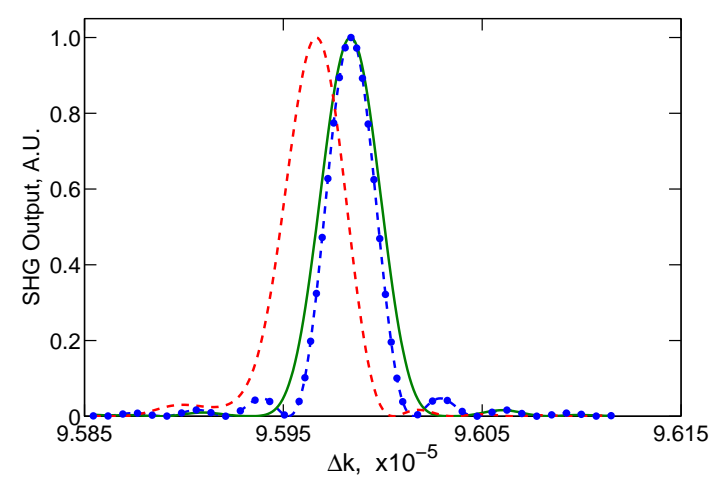

FIG. 3: Normalised $\Delta k$ tuning curves for a QPM device under plane wave (dotted curve), and focussing at $\xi=3.3$ (dashed curve), and a GQPM device with $\xi=3.3$ focussing (solid curve).

Gouy compensated quasi-phase matching (GQPM).

Theoretically, further advantages of GQPM device are the symmetric $\Delta k$ tuning response and a peak $\Delta k$ identical to a device operating under ideal plane wave conditions. This is in contrast to a standard QPM device which, as shown in Fig. (3), has an asymmetric tuning curve with a peak offset to the ideal plane wave phase matching value. This offset and the oscillations which occur at even tighter focusing are entirely due to the spatial dependence of the Gouy phase shift along the device.

GQPM must be designed for a particular focussing condition and is always most efficient with the beam waist at the centre of the crystal. In the following we examine the effect on operation for focussing at other than the design conditions or of offsetting the waist from the centre of the device. The first effect is a variation in the peak $\Delta k$ phase matching value. As can be seen in Fig.(4), GQPM has a complex relationship between focussing and peak phase matching value, with the $\Delta k$ value initially moving away from that of the plane wave before returning to zero offset at optimal focus. In contrast the peak $\Delta k$ value of a QPM device asymptotically approaches a large offset as the focus is tightened

Translation of the focussed beam along the propagation axis from a central location results in an asymmetry being re-introduced into the $\Delta k$ tuning curve as the position dependent phase adjustment of the grating no longer coincides with the centre of the Gouy phase shift. However, a similar effect is also seen in QPM when the focal position is not central to the device.

From Eq. (2) it is clear that the effects of the Gouy phase shift are further exaggerated for higher order spatial modes. The consequence of this is a rapidly evolving phase error between the fundamental wave and the grating structure, resulting in poor conversion efficiency. Thus, by specifically compensating for the high order

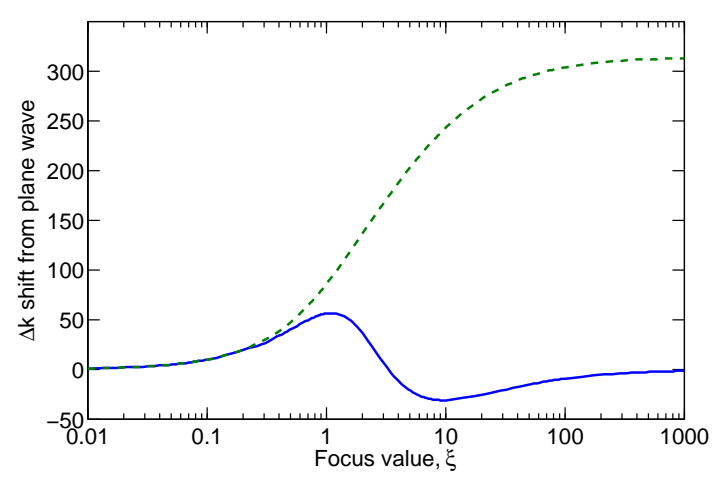

FIG. 4: A plot of the shift in peak phase matching $\Delta k$ from the plane wave case for a range of focussing values in a standard QPM structure (dashed curve) and a GQPM structure (solid).

Gouy phase large gains in efficiency should be obtained, possibly opening up the field of harmonic generation of higher order spatial modes.

In addition, the concept of compensating for a focus induced Gouy phase shift ought to be extendable to optical parametric processes, such as OPOs where the greater degree of symmetry may allow for narrower linewidths. The use of Gouy phase shift compensation to remove phase mismatch along the crystal may also help prevent parametric down conversion when attempting to achieve high conversion efficiencies in SHG.

In conclusion, we have shown that for a focussed Gaussian beam the Gouy phase shift puts a limit on the obtainable SHG conversion efficiency by preventing perfect phase matching along the length of a spatially invariant nonlinear material. We have further shown that with the use of suitable QPM devices (GQPM) the deleterious effects of the Gouy shift can in theory be completely negated, resulting in an improved efficiency of $3.5 \%$ over that predicted by BK. This higher efficiency is achieved at a tighter focussing parameter of $\xi=3.317$ than the $\xi=2.84$ value previously calculated by Boyd and Kleinman [4]. Theoretically, these devices have perfectly symmetric tuning profiles and phase matching conditions are identical to a plane wave. Finally, we have discussed the application of this result to higher order beams which could result in large efficiency gains above the $3.5 \%$ outlined here.

* hem@orc.soton.ac.uk

[1] R. Paschotta, P. Kürz, R. Henking, S. Schiller, and J. Mlynek, Opt. Lett. 19, 1325 (1994).

[2] M. Belmonte, T. Skettrup, and C. Pedersen, J. Opt. A: Pure Appl. Opt. 1, 60 (1999).

[3] L. Myers and W. Bosenberg, IEEE J. Quantum Electron. 
33, 1663 (1997)

[4] G. Boyd and D. Kleinman, J. Appl. Phys. 39, 3597 (1968).

[5] A. B. Ruffin, J. V. Rudd, J. F. Whitaker, S. Feng, and
H. G. Winful, Phys. Rev. Lett. 83, 3410 (1999). [6] S. Feng and H. G. Winful, Opt. Lett. 26, 485 (2001). 